\title{
An unusual pattern of late gadolinium enhancement (LGE) in a bi-ventricular non-compaction patient
}

\author{
Fatma Elkafrawy ${ }^{1}$, Mahmoud Abdelnabi $^{2}$, Abdelraman Assal ${ }^{1}$, and Hoda Shehata ${ }^{1}$ \\ ${ }^{1}$ Alexandria University Faculty of Medicine \\ ${ }^{2}$ Alexandria University Medical Research Institute
}

June 18, 2020

\begin{abstract}
Myocardial non-compaction (NC) is a rare genetically heterogeneous cardiomyopathy which is characterized by excessively prominent ventricular trabeculations with deep intertrabecular recesses. It is commonly believed to be due to an intrauterine arrest of the endomyocardial morphogenesis. Left ventricle (LV) is the usual site of affection, but very rarely right ventricular (RV) or both ventricles may be involved. It can be isolated or associated with other cardiac diseases or neuromuscular disorders. It has a wide spectrum of clinical manifestations that range from asymptomatic status, progressive ventricular dysfunction, arrhythmias and congestive heart failure that have been found to be associated with myocardial fibrosis. Cardiac Magnetic resonance $(\mathrm{CMR})$ is the gold standard non-invasive modality for the diagnosis of cardiomyopathies. In NC cases, late gadolinium enhancement (LGE) of the trabeculated layer or mild fibrosis of the compacted layer was previously described in the literature, however extensive fibrosis in the compacted layer only with no fibrosis at the trabeculated layer was not described before. We report a male patient presented with heart failure symptoms, echocardiogram showed severe LV and RV systolic dysfunction with prominent bi-ventricular trabeculations. CMR confirmed the diagnosis of bi-ventricular non-compaction with peculiar pattern extensive fibrosis of the compacted layer of LV in LGE. To the best of our knowledge, our case is the first case to report extensive fibrosis in the compacted layer only with no fibrosis at the trabeculated layer.
\end{abstract}

\section{Case presentation}

A 30-year-old male patient, non-diabetic, non-hypertensive, smoker presented to our facility complaining of a recent onset dyspnea grade III, orthopnea and paroxysmal nocturnal dyspnea since 3 weeks. Three days before admission he was complaining of atypical left sided chest pain and worsening of dyspnea from grade III to IV. On examination, he appeared tachypneic with bilateral congested pulsating neck veins. Vital signs, $110 / 70 \mathrm{mmHg}$, heart rate of 110 beats per minute. His laboratory tests were unremarkable. His ECG was unremarkable except for a wide QRS complex and interventricular conduction delay. Echocardiography (Figure 1, Panel A, Videos 1,2,3,4) showed dilated dimensions with global hypokinesia mostly at the septum with marked trabeculations of the apical inferior and lateral walls. Coronary angiography revealed ectatic vessels with slow flow but no obstructive lesions. CMR (Figure 1, Panel B,C,D,E,F, Videos 5,6) confirmed the presence of biventricular $\mathrm{NC}$, in the $\mathrm{LV}$, involved 11/17 segments notably the lateral wall and to a lesser extent the anterior and inferior walls. The ratio of non-compacted to compacted layer at end diastole was 4.2. Hypertrophied RV with extensive trabeculations was also noted. Marked global biventricular hypokinesia with nearly akinetic septum and very poor biventricular function $(\mathrm{LVEF}=15 \%, \mathrm{RVEF}=27 \%)$. On late gadolinium enhancement (LGE) images, extensive fibrosis was found representing $30 \%$ of the total myocardial mass. Different patterns of fibrosis were found in the form of basal/midventricular mid-wall fibrosis in 
the septum, subepicardial to mid wall fibrosis in the anterior and inferior walls (mounting to near transmural) and mid-ventricular subendocardial to mid wall fibrosis in the lateral wall as well as transmural fibrosis of all apical segments. No thrombi were found. So, the diagnosis of biventricular NC with extensive fibrosis was reported. Accordingly, the patient was maintained on anti-failure measures and anticoagulation. In our case, CMR confirmed the diagnosis of biventricular $\mathrm{NC}$ with accurate measurement of the biventricular volumes and function. It also added the presence of extensive myocardial fibrosis (which is correlated to clinical manifestations) with the ability of its quantification. The previously reported most common LGE patterns of fibrosis are subendocardial and transmural distribution in non-compacted segments and mid-wall distribution in compacted segments. ${ }^{(1,2)}$ However, in our case, there was extensive subendocardial, subepicardial, transmural and mid wall fibrosis within the compacted layer with no fibrosis at the non-compacted layer. The pathophysiological mechanisms of fibrosis in isolated NC are still uncertain. Subendocardial and transmural distribution of the non-compacted layer might be explained by the defects in the coronary microcirculation which affects the vascularization of the abnormal myocardium. $(2,3)$ The mid-wall enhancement of the compacted layer (mainly at the septum) is thought to be the result of many factors such as genetic predisposition, microvascular ischemia and the maladaptive processes related to increased wall stress caused by progressive LV remodeling that may result in focal myocyte necrosis, preferentially affecting the middle, circumferential layer. ${ }^{(4)}$

\section{Disclosure: The authors declare no conflict of interest}

\section{References:}

1. Szemraj-Rogucka Z, Majos A Left Ventricular Non-Compaction: Mid-myocardial Distribution of Late Gadolinium Enhancement in Compacted Segments. OMICS J Radiol 2017;6: 246.

2. Lachhab A, Doghmi N, Elfakir Y, Taoussi O, Benyass A, et al. (2012) Insights from magnetic resonance imaging of left ventricular non-compaction in adults of North African descent. Int Arch Med 5: 10

3. Towbin JA, Lorts A, Jefferies JL Left ventricular non-compaction cardiomyopathy. The Lancet 2015;386: 813-825.

4. Nucifora G, Aquaro GD, Pingitore A, Masci PG, Lombardi M Myocardial fibrosis in isolated left ventricular non-compaction and its relation to disease severity. Eur J Heart Fail 2011;13: 170-176.

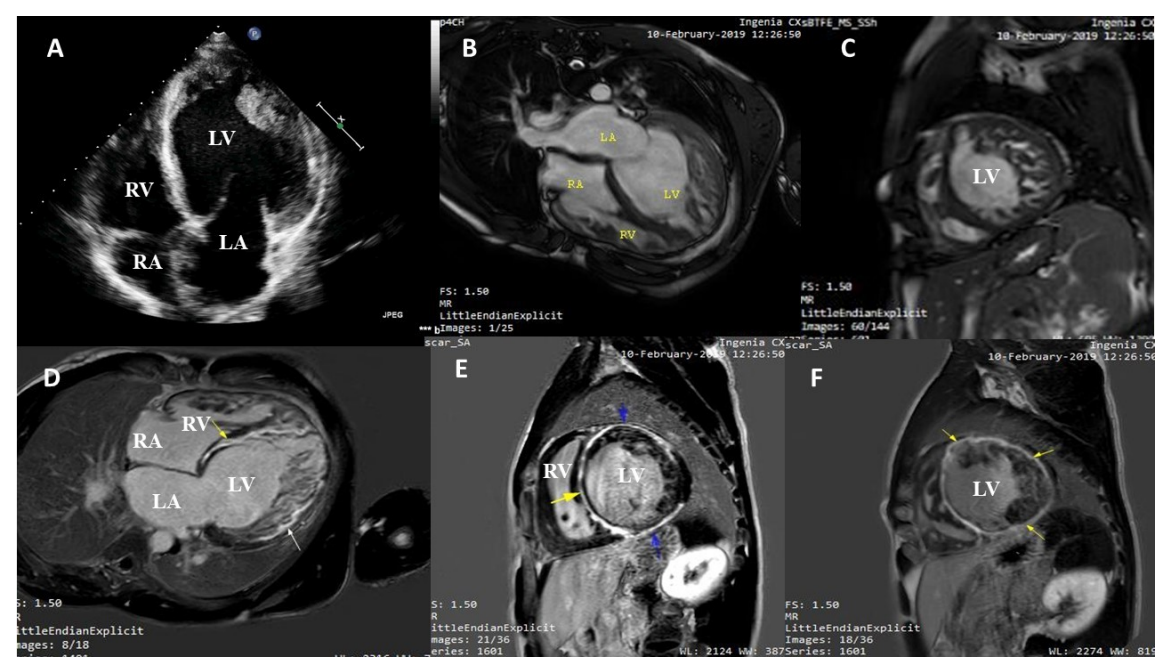

